\title{
PROGRAMAÇÃO MATEMÁTICA E A OTIMIZAÇÃO DE CUSTOS DE PRODUÇÃO
}

\author{
ANASTÁCIO PASCOAL EPANDI CANHANGA ${ }^{\mathrm{a}}$ \\ ROLANDO SOSA YBARRA ${ }^{\mathrm{b}}$ GABRIEL MÁRIO SALINGA ${ }^{\mathrm{c}}$
}

\section{Resumo}

Nos dias que correm, muitas empresas por Angola a fora desde 2015 tendem a encerrar ou reduzir sua atividade por conta da crise económica e, isto agravou-se com o surgimento da pandemia Covid-19. Chamou a atenção dos autores que a padaria do supermercado Shoprite da cidade do Cuito, Angola, deixou de produzir alguns tipos de bolos e pães que habitualmente produzia. Então resolveu-se encontrar uma maneira para minimizar os custos de produção com a Matemática, sem necessidade de deixar de produzir alguns produtos. O presente artigo tem como objetivo resolver o referido problema de redução de custos de produção na referida panificadora da cidade do Cuito. Para tal, adotou-se a programação linear como melhor forma para abordar o problema, feita a partir da interpretação dos dados fornecidos pela empresa.

Palavras-chave: Programação matemática, otimização, custos de produção

\begin{abstract}
Currently, most companies in Angola, which is a finding, since 2015 they have tended to close or reduce their business because of the economic and financial crisis has become more serious with the emergence of the covid-19 pandemic. What caught everyone's attention was the decision that the bakery at Shoprite supermarket made to stop producing certain products that were already customary for their customers. However, it was decided to find a way to reduce production costs using mathematical techniques. This article aims

anstituto Superior Politécnico do Bié, Cuito, Angola; ORCID: https://orcid.org/0000-00033255-8087E-mail: pascoalkanhanga@hotmail.com

${ }^{\text {b} U n i v e r s i d a d ~ d e ~ C i e g o ~ d e ~ A ́ v i l a-F a c u l d a d ~ d e ~ I n f o r m a ́ t i c a, ~ C i e g o ~ d e ~ A ́ v i l a, ~ C u b a ; ~ O R C I D: ~}$ https://orcid.org/0000-0003-1505-9454E-mail: rsosaybarra@gmail.com

' Instituto Superior Politécnico do Bié, Cuito, Angola; ORCID: https://orcid.org/0000-00021103-4578E-mail: gabrielsalinga@gmail.com
\end{abstract}


to solve the problem of reducing production costs of the bakery at Shoprite supermarket in the city of Cuito. For this purpose, linear programming was adopted as the best way to analyze the problem and present as another form of aid in decision making, with the interpretation of the data provided by the company.

Keywords: Mathematical programming, optimization, production cost

MSC2020: 90C05

\section{Introdução}

No presente artigo, abordou-se a programação matemática que necessáriamente movidos pela resolução de problemas reais por meio da Matemática, fez-se um estudo na padaria do supermercado Shoprite da cidade do Cuito, onde verificou-se que por adversidades do mercado vários produtos derivados do trigo deixaram de ser produzidos, pelos custos elevados da matéria prima, bem como da volatilidade do próprio mercado fruto da crise económica e depois agravada pela pandemia Covid-19. Para situar que Cuíto é uma cidade, capital da província do Bié que está no centro da República de Angola. Segundo as projeções populacionais de 2018, elaboradas pelo Instituto Nacional de Estatística, conta com uma população de 512.706 habitantes e área territorial de $4814 \mathrm{~km}^{2}$, sendo o município mais populoso da província e o décimo segundo mais populoso do país, a sua localização no território angolano é $12^{\circ} 23$ " $\mathrm{S} 16^{\circ} 56^{\prime}$ " E, a atividade principal é a agricultura, o comércio, bem como a criação de gado. Até 1975 designou-se "Silva Porto", em homenagem ao explorador português Silva Porto. Neste sentido, achou-se que a intervenção da Matemática para ajudar a tomar decisões neste campo seria uma mais valia, sendo a mesma, uma ferramenta a ter em conta no processo de tomada de decisões. Daí que, no presente artigo trazemos a tona a programação matemática pois é nela onde encontramos técnicas matemáticas com as que se pode abordar melhor o problema.

Neste contexto, fez-se uma recolha de dados a referida padaria, dados que estão relacionados com os custos, quantidades produzidas e demandas, de modos que por meio da programação linear se possa determinar quantidades aceitáveis que possam garantir um menor custo de produção a empresa, para que não seja necessário deixar de produzir alguns produtos mas que com a ideia tirada do presente resultado, o gerente possa tomar uma melhor decisão. 


\section{Programação matemática}

A programação matemática como sendo parte de Investigação de Operações (IO), é bastante prestativa no que a modelagem diz respeito na resolução de problemas das mais variadas áreas da vida produtiva e social. Nela encontramos vários modelos que subdividem a referida parte da IO que respondem à característica particular do problema que dentre outras, a programação linear, a programação não linear, programação dinâmica, programação inteira, programação mista[4]. Os problemas de programação matemática, cujo modelo[5] matemático pode ser escrito da seguinte forma:

$$
\begin{array}{r}
\text { Min(ou Max) } Z=c_{1} x_{1}+c_{2} x_{2}+\cdots+c_{n} x_{n} \\
\text { Sujeito } a: a_{11} x_{1}+a_{12} x_{2}+\cdots+c_{1 n} x_{n} \leq b_{1} \\
a_{21} x_{1}+a_{22} x_{2}+\cdots+c_{2 n} x_{n} \leq b_{2} \\
\vdots \quad \vdots \quad \vdots \\
\vdots \quad \vdots \quad x_{m 1} x_{1}+a_{m 2} x_{2}+\cdots+c_{m n} x_{n} \leq b_{m} \\
x_{1} x_{2} \cdots x_{n} \geq 0
\end{array}
$$

problemas de programação inteira

$$
\begin{array}{r}
\operatorname{Max}\left(\text { ou Min) } Z=c_{1} x_{1}+c_{2} x_{2}+\cdots+c_{n} x_{n}\right. \\
\text { Sujeito } a: a_{11} x_{1}+a_{12} x_{2}+\cdots+c_{1 n} x_{n} \leq b_{1} \\
a_{21} x_{1}+a_{22} x_{2}+\cdots+c_{2 n} x_{n} \leq b_{2} \\
\vdots \quad \vdots \quad \vdots \\
a_{m 1} x_{1}+a_{m 2} x_{2}+\cdots+c_{m n} x_{n} \leq b_{m} \\
x_{j} \in Z \forall j=1,2, \cdots, p(\leq n) \\
x_{j} \geq 0 \forall j=p+1, \cdots, n
\end{array}
$$

Neste tipo de programação, todas as variáveis só podem ser inteiras, as quais representam decisões, quando não forem todas inteiras, então já estaremos diante de outro tipo de problema de programação que seria programação inteira mista. 


\section{Programação Linear}

A programação linear, a podemos definir como sendo uma técnica matemática que permite otimizar (maximizar ou minimizar) uma função linear designada função objetivo, a qual fica sujeita a determinadas limitações e/ou disponibilidades a que designamos por restrições.

A função objetivo habitualmente é escrita como sendo a soma dos produtos do custo pela quantidade [1],

$$
\sum_{j}^{n} c_{n} x_{n}
$$

como é a função da expressão (1). E representa a meta que se pretende atingir, a otimização de custos ou prejuízos (minimização) e de lucros ou benefícios (maximização).

As restrições são inequações ou equações que representam as limitações e/ou disponibilidades a que a função objetivo está sujeita, ou seja, as condições que intervêm na prossecussão de uma determinada atividade a qual o problema identificado é que formam as inequações (2), (3) e (5) e, a restrição (6) é de não negatividade, nesta restrição assumimos que as variáveis de decisão são não negativas[1, p. 27-29].

\subsection{Métodos de resolução}

Para resolver problema de programação linear do modelo exposto anteriormente, tradicionalmente usam-se o Método Gráfico (contudo, este método não é usual na resolução de problemas do cotidiano), que por meio da representação gráfica das restrições é possível esboçar a região de soluções fatíveis e no entanto, determinar a solução ótima do problema, de salientar que como os outros métodos, este também tem suas vantagens e limitações. E o algorítmo de Simplex com as suas variantes [2], [3]. Isto quer dizer que, quando o problema de programação linear, seu modelo matemático estiver na forma canónica, será resolvido utilizando o algorítmo do Simplex Primal e se terá utilizado as variantes do Método Simplex (Método de duas fases e Método M-Grande) caso o modelo matemático de programação linear estiver descanonizado. Forma canónica é uma maneira codificada e única de descrever um ente matemático, ganhando assim um padrão universal[6], o método simplex primal e suas variantes são métodos iterativos que determinam numericamente a solução ótima, encontramos diferença na abordagem, já que no simplex de duas fases procura-se minimizar a função objectivo auxiliar e tornar as variáveis artificiais não básicas, enquanto no simplex M-grande, aplica-se uma penalização nas variáveis 
artificiais [6] e [7]. A programação matemática é utilizada para resolução de problemas das mais variadas áreas, sobretudo em empresas, é de salutar importância o emprego do computador para resolver com rapidez e dar efeciência a tomada de decisão. Neste sentido, para resolver um problema de programação linear podemos utilizar o suplemento do Excel, o Solver ou ainda um software de linguagem de programação como é o Matlab, Octave, R, entre outros. Mas para resolver o problema que nos propusemos aqui, utilizaremos o Octave já que é um software gratuíto.

\section{Modelação do problema}

Tabela 1: Custos de matéria-prima

\begin{tabular}{ccc}
\hline Designação & Quantidades & Preços (em kwanzas) \\
\hline Farinha de trigo & $50 \mathrm{~kg}$ & $10.000,00$ \\
Fermento & $0,5 \mathrm{~kg}$ & $1.000,00$ \\
Leite & $2 \mathrm{Litros}$ & $1.000,00$ \\
Ovos & $30 \mathrm{ovos}$ & $3.799,85$ \\
Sal genérico & 500 gramas & 600,00 \\
Acúcar genérico & $1 \mathrm{~kg}$ & 700,00 \\
Melhorante & $0,5 \mathrm{~kg}$ & 500,00 \\
Óleo de Cozinha & $1 \mathrm{Litro}$ & $1.000,00$ \\
Vinagre pasteleiro & $150 \mathrm{ml}$ & 400,00 \\
\hline
\end{tabular}

Fonte: Elaborado pelos autores com base os dados fornecidos pela empresa.

Com base nas informações recolhidas na padaria do supermercado Shoprite da cidade do Cuito e as que espelha a tabela 1, deduziu-se os seguintes dados apresentados na tabela 2:

A questão é saber as quantidades que devem ser produzidas para que o custo seja mínimo.

Para modelar o problema apresentado, definiram-se variáveis as seguintes:

$x_{1}=$ quantidade de pão francês;

$x_{2}=$ quantidade de bolo rainha;

$\mathrm{e}$

$x_{3}=$ quantidade de pão de avó.

Com o exposto anteriormente, foi possível extrair o seguinte modelo matemático de programação linear: 
Tabela 2: Quantidades de ingredientes por unidade de produto.

\begin{tabular}{|c|c|c|c|c|}
\hline Ingredientes & Pão francês & Bolo rainha & Pão de avó & Disponibilidade \\
\hline Farinha de trigo & $0.18 \mathrm{~kg}$ & $0.06 \mathrm{~kg}$ & $0.25 \mathrm{~kg}$ & $\mathbf{5 0 0 0} \mathbf{~ k g}$ \\
\hline Sal genérico & $0.002 \mathrm{~kg}$ & $0.004 \mathrm{~kg}$ & - & $\mathbf{1 1 5} \mathbf{~ k g}$ \\
\hline Fermento & $0.002 \mathrm{~kg}$ & $0.003 \mathrm{~kg}$ & $0.003 \mathrm{~kg}$ & $\mathbf{8 7 \mathbf { k g }}$ \\
\hline Leite & $0.007 \mathrm{l}$ & $0.03 \mathrm{l}$ & - & $\mathbf{2 5 1} \mathbf{~}$ \\
\hline Ovo & - & $10 \mathrm{gr}$ & - & $\mathbf{9 5} \mathbf{~ e m b a l a g e n s}$ \\
\hline Acúcar genérico & $0.032 \mathrm{~kg}$ & $0.012 \mathrm{~kg}$ & - & $\mathbf{2 2 6} \mathbf{~ k g}$ \\
\hline Óleo de cozinha & $0.004 \mathrm{l}$ & $0.03 \mathrm{l}$ & $0.007 \mathrm{l}$ & $\mathbf{1 2 6} \mathbf{l}$ \\
\hline Vinagre & $0.0005 \mathrm{l}$ & - & $0.012 \mathrm{l}$ & $\mathbf{1 4 8} \mathbf{l}$ \\
\hline Demanda & $\mathbf{7 0 0} \mathbf{u n i d a d e s}$ & $\mathbf{1 8 0} \mathbf{u n i d a d e s}$ & $\mathbf{1 0} \mathbf{u n i d a d e s}$ & \\
\hline Custo de produção & $\mathbf{4 8 . 9} \mathbf{~ k z}$ & $\mathbf{1 1 7} \mathbf{~ k z}$ & $\mathbf{1 0 7} \mathbf{~ k z}$ & \\
\hline
\end{tabular}

Fonte: Elaborado pelos autores com base os dados fornecidos pela empresa.

$$
\text { Minimizar } Z=48,9 x_{1}+117 x_{2}+107 x_{3}
$$

Sujeito a:

$$
\begin{array}{r}
0,18 x_{1}+0,06 x_{2}+0,25 x_{3} \leq 5000 \\
0,002 x_{1}+0 x_{2}+0,004 x_{3} \leq 115 \\
0 x_{1}+10 x_{2}+0 x_{3} \leq 95 \\
0,002 x_{1}+0,003 x_{2}+0,003 x_{3} \leq 87 \\
0,004 x_{1}+0,03 x_{2}+0,007 x_{3} \leq 126 \\
0,007 x_{1}+0,03 x_{2}+0 x_{3} \leq 251 \\
0 x_{1}+0,032 x_{2}+0,012 x_{3} \leq 226 \\
0,0005 x_{1}+0 x_{2}+0,012 x_{3} \leq 148 \\
x_{1}+0 x_{2}+0 x_{3} \geq 700 \\
0 x_{1}+x_{2}+0 x_{3} \geq 180 \\
0 x_{1}+0 x_{2}+x_{3} \geq 10 \\
x_{1}, x_{2}, x_{3} \geq 0
\end{array}
$$

Neste modelo temos as restrições da farinha de trigo (8), Sal genérico (9), Ovos (10), fermento (11), óleo de cozinha (12), leite (13), acúcar (14), vinagre (15) e as restrições de demanda de cada produto tais como: demanda do pão francês (16), 
demanda do bolo rainha (17) bem como demanda do pão de avó (18), também temos a restrição de não negatividade (19), que na verdade, como as variáveis representam quantidades concretas, não devem ser negativas.

Este problema levaria muito tempo para resolvê-lo manualmente utilizando o algorítmo de Simplex, daí que para resolver em pouco tempo deve-se utilizar o computador, neste caso, recorremos ao Octave que é uma linguagem de programação, também compatível com o Matlab e determinou-se a solução do problema.

Para a construção da rotina que nos leva a solução ótima, primeiro devemos evidenciar as matrizes que compõem o problema:

$c=\left[\begin{array}{c}48,9 \\ 117 \\ 107\end{array}\right], A=\left[\begin{array}{ccc}0,18 & 0,06 & 0,25 \\ 0,002 & 0 & 0,004 \\ 0 & 10 & 0 \\ 0,002 & 0,003 & 0,003 \\ 0,004 & 0,03 & 0,007 \\ 0,007 & 0,03 & 0 \\ 0 & 0,032 & 0,012 \\ 0,0005 & 0 & 0,012 \\ 1 & 0 & 0 \\ 0 & 1 & 0 \\ 0 & 0 & 1\end{array}\right]$ e $b=\left[\begin{array}{c}5000 \\ 115 \\ 95 \\ 87 \\ 126 \\ 251 \\ 226 \\ 148 \\ 700 \\ 180 \\ 10\end{array}\right]$

e também a matriz das variáveis

$x=\left[\begin{array}{l}x_{1} \\ x_{2} \\ x_{3}\end{array}\right]$

O que nos resulta o modelo:

$\operatorname{Min} Z=c^{t} x$

Sujeito a:

$$
\begin{array}{r}
A x \leq \geq b \\
x_{1}, x_{2}, x_{3} \geq 0
\end{array}
$$

Onde $c$ é a matriz dos custos, os coeficientes que aparecem na função objetivo, a 
matriz $A$ é a matriz dos coeficientes do membro esquerdo das restrições, enquanto que $b$ é a matriz dos termos independentes nas restrições. O problema foi resolvido usando uma função interna do Octave, a glpk, como se pode observar na figura 1 que segue:

Figura 1: Programa de resolução do Problema de Programação Linear

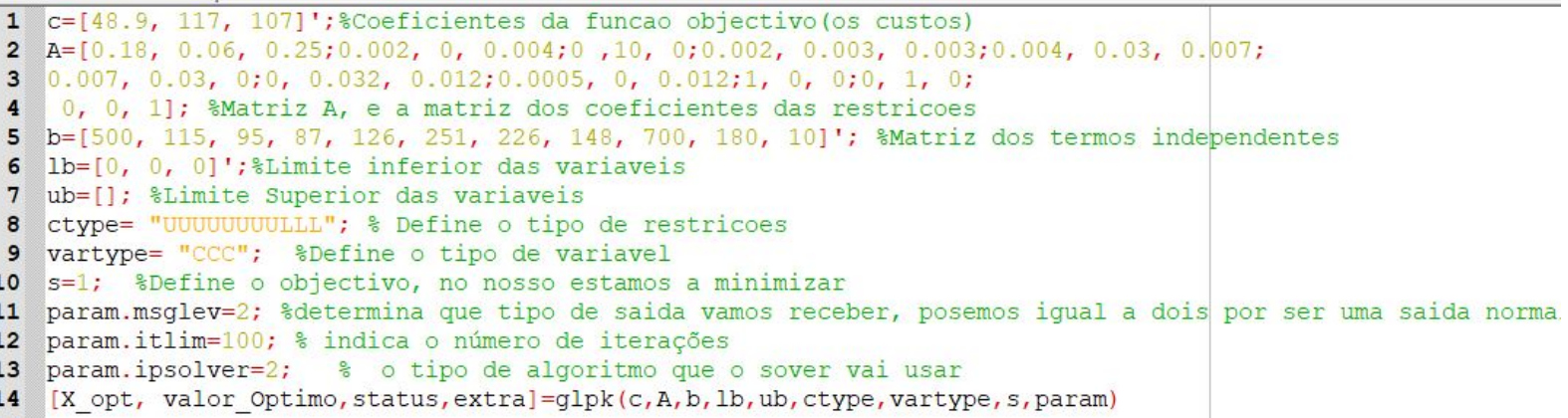

Fonte: Elaborado pelos autores através do Octave.

A tabela 3, mostra o resultado encontrado para o nosso problema, interessa aqui tanto o valor óptimo da função bem como os valores ótimos para as variáveis.

Tabela 3: Resultados do problema.

\begin{tabular}{|c|c|c|c|c|}
\hline Variáveis & $x_{1}$ & $x_{2}$ & $x_{3}$ & $\mathrm{Z}$ \\
\hline Resultados & 700 & 9.5 & 10 & $3.6412 \mathrm{e}+004$ \\
\hline
\end{tabular}

Como se pode observar na tabela 3 , o melhor custo que se pode ter com a fabricação dos três produtos é 36.411, 5 kwanzas, olhando para o problema em concreto e os resultados para a produção do o pão francês, bolo rainha e pão de avó respectivamente são $(700 ; 9,5 ; 10)$, no entanto, por uma questão de coerência o 9.5 deve ser arredondado porque refere-se a bolos. Podemos observar também que os números referentes a produção do pão francês e do pão de avó coincidem com as quantidades que a empresa apresenta como demanda, de acordo com o gerente, eles determinam as mesmas por via do fluxo diário da procura de cada produto. 


\section{Conclusão}

A Matemática oferece-nos bastantes ferramentas que podem ser utilizadas para garantir uma melhor decisão às empresas e não só. O problema apresentado foi resolvido fazendo uso da programação matemática e espelha que é possível determinar as melhores quantidades que ofereçam menor custo para a partir daí o gestor tomar a decisão que se aproxime mais à realidade.

\section{Referências}

[1] HILLIER, F. S.; LIEBERMAN, G. J.. Introduccion a la investigación de operaciones. 5ta. edición. Novena edición ISBN: 978-607-15-0308-4 (ISBN edición anterior: 970-10-5621-3), 2010.

[2] EBRAHIMNEJAD, A.; NASSERI, S. H.; LOTFI, F. H.; SOLTANIFAR, M.. A primal-dual method for linear programming problems with fuzzy variables. European J. Industrial Engineering, vol. 4, No. 2, 2010.

[3] COSMIS, De S.; LEONE, De R.. The use of grossone in Mathematical Programming and Operating Research. Applied Mathematics and Computation, Vol 218, Issue 16, 15 April 2012, Pages 8029-8038.

[4] DANTZIG, G. B. (2002) Linear Programming. Operations Research 50(1):4247. https://doi.org/10.1287/ opre.50.1.42.17798

[5] CANHANGA, A. E. P. (2020) Modelo matemático para previsão de vendas: Regressão linear simples. Cadernos do IME - Série Matemática, Rio de Janeiro, n. 14, 2020, p. 55-65 - Doi: 10.12957/cadmat.2020.50427

[6] SILVA, C. T. L.; ARENALES, M. N.. Métodos tipo dual simplex para problemas de optimização linear canalizados e esparsos. Pesquisa Operacional, v.27, n.3, p.457-486, Setembro a Dezembro de 2007

[7] SILVA, A. C. M.; SANTANA, C. F. D.; BRITO, V. C.; SIlVA, E. C.; RIBEIRO, R. R. F.; PEREIRA, G. E. S. Otimização do sistema de abastecimento de água da Barragem de Jucazinho através de programação linear. Revista IberoAmericana de ciências ambientais. v.10-n.5. Ago a Set 2019. 